\title{
Spinal epidural abscess complicating chemotherapy in patients with leukemia
}

\author{
Laura Moschettoni, M.D., Agazio Menniti, M.D., Giovanni Liccardo, M.D., \\ and Pierpaolo LunARdi, M.D.
}

Department of Neurosciences Neurosurgery, University of Rome “Tor Vergata,” Rome, Italy

\begin{abstract}
The authors reviewed the basic facts about infections that complicate chemotherapy, and found that a difference exists between those that are medically treated, for which several standard protocols have been adopted, and infections amenable to surgical treatment, such as spinal epidural abscess, which are managed according to the experience at individual institutions. The authors believe that patients with leukemia who manifest a spinal epidural abscess should always be surgically treated when the infection occurs between the induction and remission phases, whereas medical treatment options can be considered for spinal epidural abscesses occurring at the end of the chemotherapy course.
\end{abstract}

\section{KeY Words • spinal epidural abscess • infection • chemotherapy $\bullet$ leukemia}

Spinal epidural abscess is a rare ${ }^{9}$ and potentially lifethreatening condition, with a very high morbidity rate and an overall mortality rate of 10 to $20 \%{ }^{2,3,29}$ In the largest series, investigators have stressed the importance of early diagnosis in providing adequate treatment, ${ }^{15,19}$ be it surgical decompression or conservative management.

Generally accepted risk factors for spinal epidural abscess are conditions of systemic immunocompromise such as human immunodeficiency virus, diabetes mellitus, alcoholism, and long-term corticosteroid treatment. ${ }^{7,22,25} \mathrm{~Pa}$ tients undergoing cancer treatment have also been recognized as an at-risk group ${ }^{25,29}$ for this pathological entity. Leukemia is not a rare condition; its incidence is 9.6 per 100,000 population per year (including both acute and chronic forms), and we believe that identification of basic guidelines for surgical treatment of infections in patients with leukemia would result in a better and safer treatment.

\section{Infections in Patients With Leukemia}

Infections related to immune system impairment occur in 60 to $100 \%$ of patients receiving high-dose chemotherapy and are the most frequent complication of treatment in patients with leukemia; they remain the principal cause of morbidity and mortality in $75 \%$ of these patients. During chemotherapy, the bone marrow of patients with leukemia undergoes profound and often prolonged periods of druginduced myelosuppression (neutropenia for $>4$ weeks). Antineoplastic agents, radiation therapy, and corticosteroid drugs all play a role ${ }^{23}$ in promoting this condition, the first disrupting chemotaxis and phagocytosis and compromising the neutrophils' ability to eliminate intracellular microorganisms, whereas the other two contribute to delays in recovery of peripheral blood neutrophil counts.

Abbreviation used in this paper: $\mathrm{MR}=$ magnetic resonance.

\section{Medical Treatment of Infectious Complications}

A sudden drop in the peripheral neutrophil count $(<$ 150 cells/ $\mu \mathrm{l}$ ) leaves patients vulnerable to life-threatening bacterial, viral, and fungal infections, ${ }^{8,23,24}$ and the outcome depends on early initiation of appropriate therapy. Unfortunately, prompt diagnosis of infection is difficult because early signs and symptoms may be absent or nonspecific, and laboratory and neuroimaging findings frequently are unremarkable. To complicate the issue, immunosuppressed patients may have multiple infections during a single clinical presentation. Therapy is often instituted empirically, based on the clinical presentation and symptoms. Initial therapy in patients with neutropenia who present with fever should cover the most common and life-threatening pathogens (Table 1).

The spectrum of the empirical antimicrobial treatment has to include Gram-negative aerobic bacteria ${ }^{4,7}$ (Enterobacteriaceae and Pseudomonas aeruginosa) and Grampositive cocci (streptococci, enterococci, and Staphylococcus aureus), and should be adapted to local resistance. Initial therapy does not necessarily have to cover coagulase-negative staphylococci, oxacillin-resistant $S$. aureus, anaerobic bacteria, and fungi. In case of treatment failure (persistent fever or clinical instability), second-line therapy should include a glycopeptide, teicoplanin, or vancomycin, and should close antimicrobial activity gaps in the Gram-negative spectrum. If patients are clinically stable, a switch to second-line therapy should not be made earlier than 96 hours after the start of treatment, because some patients are slow to respond. Patients with an expected duration of neutropenia of more than 7 days and in whom clinical deterioration is observed should also be given parenteral antifungal therapy with amphotericin B or fluconazole.

Most infectious complications of medical interest in 
TABLE 1

Pathogens and clinical syndromes in patients with spinal infections

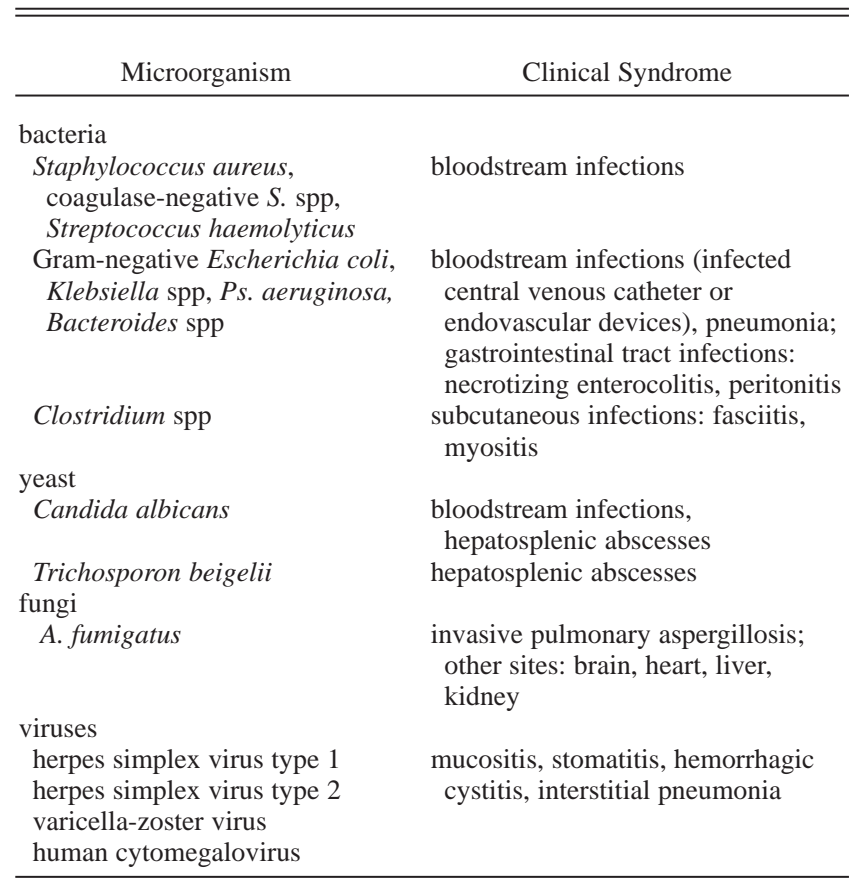

patients with leukemia who are undergoing chemotherapy can be effectively managed. Both prophylaxis, accomplished by following the rules of hygiene and by administering routinely used antibiotics, and early recognition of the disease are essential for effective treatment.

\section{Surgical Management of Infectious Complications}

Management of infections requiring surgical treatment in patients with leukemia is not as codified as is medical therapy. In the literature, cases of infections of anorectal regions, small bowel obstruction after chemotherapy, lifethreatening toxic megacolon, perforation of the bowel, and necrotizing enterocolitis have been reported. . $^{10,16,17,28}$ Antineoplastic agents such as etoposide or cytosine arabinoside, which are associated with severe gastrointestinal mucosal excoriation, increase the risk of some of these complications. The new purine analogs ${ }^{1,21,26}$ have been associated with opportunistic infections caused by uncommon microorganisms such as Pneumocystis carinii, Listeria monocytogenes, and fungi. Clinical parameters often are subtle, but high-grade unresponsive fever, abdominal pain, and diarrhea must be evaluated promptly.

In the literature we could not identify guidelines for surgical treatment of infections in patients with leukemia: the cases reported all highlight the lack of a standard protocol and the importance of the experience at a particular institution in handling such situations. Some agree on the desirability, when possible, of delaying surgery ${ }^{23}$ until neutrophil counts improve, because recovery of functional neutrophil levels has a favorable impact on outcome. This requires a close monitoring of the complete blood count and infectious markers, which is not straightforward in patients with leukemia, whose blood parameters are all out of the normal ranges.

Antineoplastic drugs have a very strong impact on the human immune system, and all cause different levels of myelosuppression, depending on their mechanism of action and target. Standard induction therapy in leukemia typically involves either a four-drug regimen of vincristine, prednisone, anthracycline, and cyclophosphamide or Lasparaginase, or a five-drug regimen of vincristine, prednisone, anthracycline, cyclophosphamide, and L-asparaginase given over the course of 4 to 6 weeks. Consolidation therapy includes cytosine arabinoside in combination with anthracycline or epipodophyllotoxin. ${ }^{13,14}$ Chemotherapeutic treatment of leukemia, compared with other malignancies such as breast or lung cancer, ${ }^{5,18}$ might be continued for a longer period of time, up to 2 years, and we believe that this peculiarly massive and prolonged immunosuppression renders patients with leukemia more exposed to dangerous infectious complications.

\section{Spinal Epidural Abscess in Patients With Leukemia}

Spinal epidural abscess complicating the chemotherapeutic course is a rarely described occurrence. In a review of the literature we identified a few cases of spinal infectious complications in patients receiving antileukemia treatment. Cases of spinal infections caused by opportunistic agents such as Pn. carinii, L. monocytogenes, and fungi are rare, as is vertebral osteomyelitis caused by $A s$ pergillus fumigatus: only 39 cases have been described between 1966 and 1998, most of them occurring in patients with immune impairment. ${ }^{27}$

Clinical presentation of spinal epidural abscess in immunocompromised patients might not include the classic triad of fever, high white blood cell count, and high erythrocyte sedimentation rate in laboratory tests as well as signs of radiculopathy or myelopathy. Initial signs and symptoms can be subtle, consisting of localized spinal pain, and later signs of myeloradicular compression might occur: these include radicular pain, paresthesias, muscle weakness, sensory loss, sphincter dysfunction, and finally paralysis.

Magnetic resonance imaging is the method of choice for diagnosis of spinal epidural abscess; Gd-enhanced MR images can aid in the categorization of the age and consistency of the lesion (that is, pus or granulation tissue), and this modality is helpful in defining the relationship of the abscess to nerve structures, revealing evidence of spinal cord compression, although no relationship between signs of compression on MR images and the rate of disease progression has been proved. Notably, MR imaging findings are helpful in planning appropriate interventions: ${ }^{20}$ a solid appearance of granulation tissue requires a more extended laminectomy, whereas imaging findings of areas of liquid pus indicate the feasibility of limited laminotomies and washout of the epidural space to treat the abscess.

Spinal epidural abscess can be treated either surgically or conservatively. Indications for surgical treatment ${ }^{12}$ include progressive neurological deficit, persistent severe pain, or persistent fever and leukocytosis despite adequate antibiotic therapy. We believe that in patients with leu- 


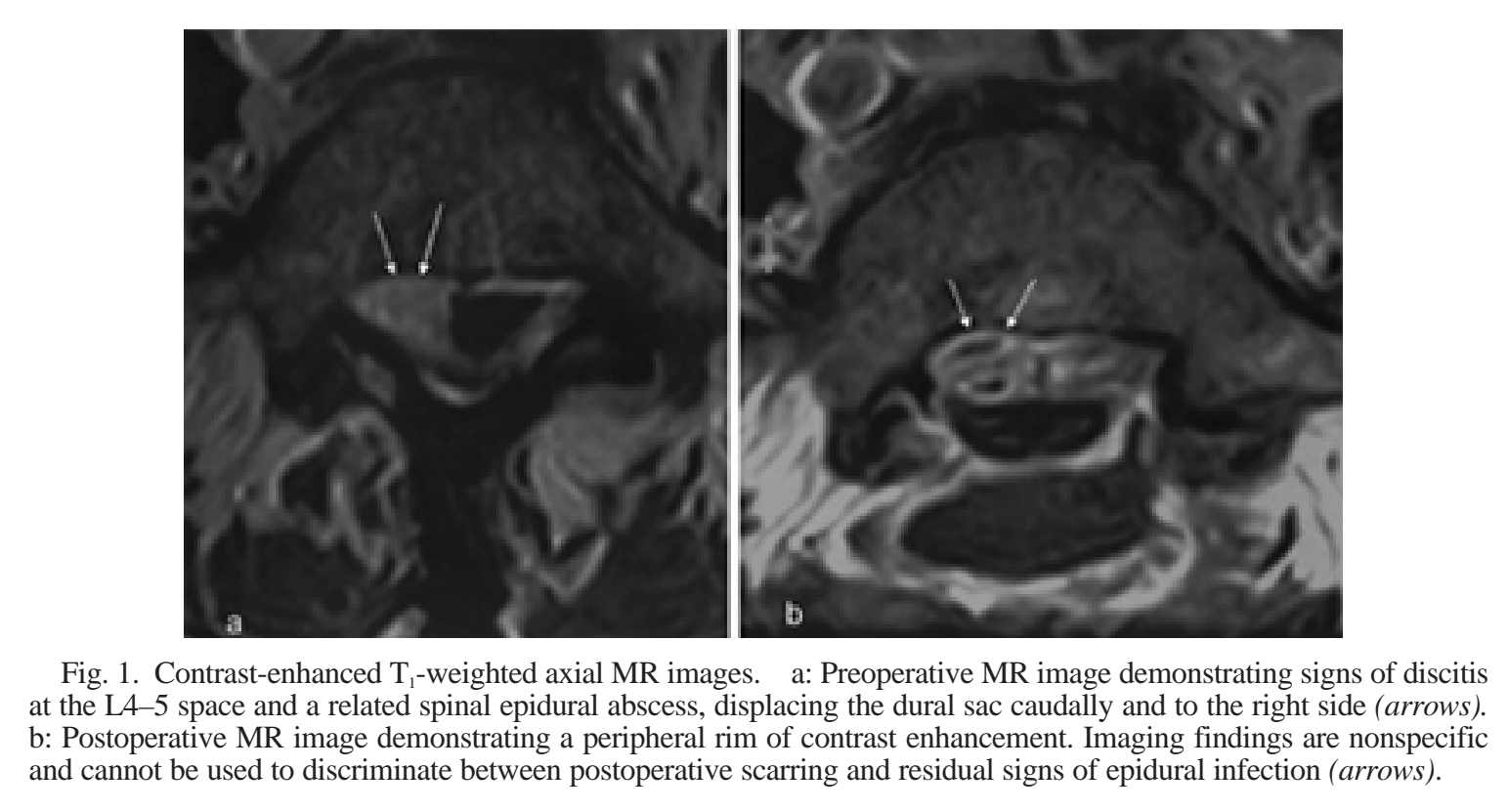

kemia the main concern is the timing of treatment: when rather than how to treat the abscess. In fact, the bone marrow of a patient with leukemia typically requires 2 to 3 weeks to recover after standard induction therapy, and postremission therapy is immediately initiated to prevent recurrence of leukemia and development of drug resistance.

We believe that a spinal epidural abscess occurring between the first and second phase of chemotherapy leaves no time to evaluate the effectiveness of antibiotic therapy, because failure of conservative management would result in a deferred intervention and in a delayed start of the second-phase consolidation chemotherapy, favoring relapse of leukemia. In our opinion a spinal epidural abscess developing after induction of chemotherapy should be surgically drained in any case: the choice of an adequate surgical approach is then mandatory to compromise between successful drainage of the abscess and minimally invasive surgery, especially for multilevel, extended abscesses. We believe granulation tissue that is tightly adherent to the dura mater should not be removed if this involves a risk of fissure in the dura mater itself, because spread of infection could occur through the cerebrospinal fluid.

We think that selection of appropriate treatment of spinal epidural abscess in patients with leukemia must be adjusted in relation to their chemotherapy schedule and to the presence of motor deficit. After surgery, consolidation chemotherapy should be initiated only when the infection has completely resolved. This might be difficult to prove because infection markers are quite out of range in these cases, and only a limited period of observation is possible before continuing chemotherapy. Careful monitoring of the patient's clinical condition and results of laboratory tests is mandatory.

Follow-up MR images can be useful in determining residual signs of infection, but postoperative findings are quite nonspecific, because a contrast-enhanced area might represent either postoperative scarring or a residual pus collection (Figs. 1 and 2). A bone scan performed with gallium contrast at 6 weeks, if negative, might indicate that infection had resolved. ${ }^{11}$ We believe that results of clinical examination together with laboratory tests and imaging findings should be considered in the postoperative follow-up review. Motor deficits improve slowly after surgical decompression, but lessening of preoperative pain could indicate resolution of epidural infection. When spinal epidural abscess occurs after the last chemotherapy treatment, the standard protocol currently adopted in the literature can be considered.

\section{CONCLUSIONS}

Standard guidelines for surgical management of these infections have not yet been identified, but there is a gen-

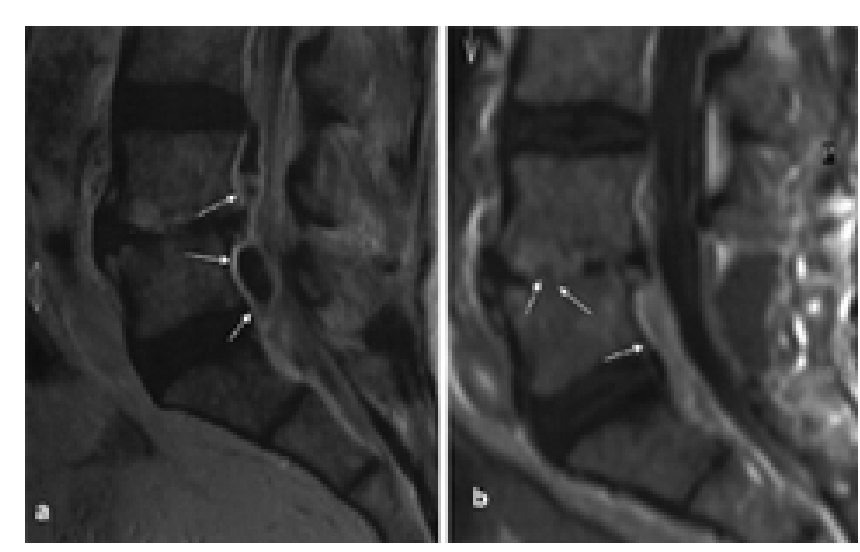

Fig. 2. Contrast-enhanced $\mathrm{T}_{1}$-weighted sagittal MR images; same patient. a: Preoperative MR image demonstrating craniocaudal extension of the epidural collection, limited to the L4-5 interspace (arrows). b: Postoperative MR image obtained at the 3-week follow-up review documenting the surgical access point and areas of nonspecific and inhomogeneous contrast enhancement (arrows), despite the patient's clinical improvement. 
eral agreement that a close collaboration between the hematologist and the surgeon may provide guidelines for therapy in such cases, giving these patients the possibility of survival and the opportunity to continue the treatment planned for the primary disease.

\section{References}

1. Anaissie EJ, Kontoyiannis DP, O'Brien S, et al: Infections in patients with chronic lymphocytic leukemia treated with fludarabine. Ann Inter Med 129:559-566, 1998

2. Baker AS, Ojemann RG, Baker RA: To decompress or not to decompress-spinal epidural abscess. Clin Infect Dis 15: 28-29, 1992

3. Baker AS, Ojemann RG, Swartz MN, et al: Spinal epidural abscess. N Engl J Med 293:463-468, 1975

4. Bertz H, Auner HW, Weissingerl F, et al: Antimicrobial therapy of febrile complications after high-dose chemo-/radiotherapy and autologous hematopoietic stem cell transplantationguidelines of the Infectious Diseases Working Party (AGIHO) of the German Society of Hematology and Oncology (DGHO). Ann Hematol 82 (Suppl 2):S167-S174, 2003

5. Burstein HJ, Bunnell CA, Winer EP: New cytotoxic agents and schedules for advanced breast cancer. Semin Oncol 28: 344-358, 2001

6. Capria S, Vitolo D, Cartoni C, et al: Neutropenic enterocolitis in acute leukemia: diagnostic and therapeutic dilemma. Ann Hematol 83:195-197, 2004

7. Cornely OA, Hiddemann W, Link H, et al: Interventional antimicrobial therapy in febrile neutropenic patients-Paul Ehrlich Society for Chemotherapy PEG study II. Ann Hematol 74 (Suppl 1):A51, 1997 (Abstract)

8. De Pauw BE, Donnelly JP: Infections in the immunocompromised host: general principles, in Mandell GL, Bennett JE, Dolin R (eds): Mandell, Douglas, and Bennett's Principles and Practices of Infectious Diseases, ed 5. Philadelphia: Churchill Livingstone, 2000, pp 3079-3090

9. Del Curling O Jr, Gower DJ, McWorther JM: Changing concepts in spinal epidural abscess: a report of 29 cases. Neurosurgery 27:185-192, 1990

10. Dosik GM, Luna M, Valdivieso M, et al: Necrotizing colitis in patients with cancer. Am J Med 67:646-656, 1979

11. Hadjipavlou AG, Bergquist SC, Chen JW, et al: Vertebral osteomyelitis. Curr Treat Op Infect Dis 2:226-237, 2000

12. Harrington P, Millner PA, Veale D: Inappropriate medical management of spinal epidural abscess. Ann Rheum Dis 60: 218-222, 2001

13. Hoelzer D: Acute lymphoblastic leukemia in adults, in Hoffman R, Benz EJ (eds): Hematology: Basic Principles and Practice. Philadelphia: Churchill Livingstone 1991, pp 793-804

14. Hoelzer D, Gökbuget N, Ottmann O, et al: Acute lymphoblastic leukemia. Hematology:162-192, 2002
15. Khanna RK, Malik GM, Rock JP, et al: Spinal epidural abscess: evaluation of factors influencing outcome. Neurosurgery 39: 958-964, 1996

16. Koea JB, Shaw JH: Surgical management of neutropenic enterocolitis. Br J Surg 76:821-824, 1989

17. Lehrnbecher T, Marshall D, Gao C, et al: A second look at anorectal infections in cancer patients in a large cancer institute: the success of early intervention with antibiotics and surgery. Infection 30:272-276, 2002

18. Leslie WT, Bonomi PD: Novel treatments in non-small cell lung cancer. Hematol Oncol Clin North Am 18:245-267, 2004

19. Mackenzie AR, Laing RB, Smith CC, et al: Spinal epidural abscess: the importance of early diagnosis and treatment. J Neurol Neurosurg Psychiatry 65:209-212, 1998

20. Parkinson JF, Sekhon LHS: Surgical management of spinal epidural abscess: selection of approach based on MRI appearance. J Clin Neurosci 11:130-133, 2004

21. Perkins JG, Flynn JM, Howard RS, et al: Frequency and type of serious infections in fludarabine-refractory B-cell chronic lymphocytic leukemia and small lymphocytic lymhoma: implications for clinical trials in this patient population. Cancer 94: 2033-2039, 2002

22. Rigamonti D, Liem L, Sampath P, et al: Spinal epidural abscess: contemporary trends in etiology, evaluation, and management. Surg Neurol 52:189-197, 1999

23. Safdar A: Managing opportunistic infections against the odds of neutropenia: drug-induced myelosuppression sets the stage. J Crit Illness 18:54-60, 2003

24. Safdar A, Armstrong D: Infectious morbidity in critically ill patients with cancer. Crit Care Clin 17:531-570, vii-viii, 2001

25. Sampath P, Rigamonti D: Spinal epidural abscess: a review of epidemiology, diagnosis, and treatment. J Spinal Disord 12: 89-93, 1999

26. Santos AB, Llamas P, Gadea I, et al: Aspergillus fumigatus: a rare cause of vertebral osteomyelitis. Haematologica 89: ECR10, 2004

27. Vinas FC, King PK, Diaz FG: Spinal aspergillus osteomyelitis. Clin Infect Dis 28:1223-1229, 1999

28. von Lilienfeld-Toal M, Ebert O, Theuerkauf I, et al: Small bowel obstruction in acute myelogenous leukemia: stenosis or paralysis? Ann Hematol 80:611-613, 2001

29. Wessling H, de las Heras P: Cervicothoracolumbar spinal epidural abscess with tetraparesis. Good recovery after non-surgical treatment with antibiotics and dexamethasone. Case report and review of literature. Neurocirugia 14:529-533, 2003

Manuscript received October 15, 2004.

Accepted in final form November 10, 2004.

Address reprint requests to: Pierpaolo Lunardi, M.D., Department of Neurosciences Neurosurgery, University of Rome, Tor Vergata, Via Montpellier 1, 00133 Rome, Italy. email: lunardi@ med.uniroma2.it. 